Madsen, T. B., and A .F. Swann. (????) "Toric geometry of Spin(7)-manifolds,"

International Mathematics Research Notices, Vol. ????, Article ID ????, 17 pages.

doi:10.1093/imrn/????

\title{
Toric geometry of Spin(7)-manifolds
}

\section{Thomas Bruun Madsen ${ }^{1}$ and Andrew Swann ${ }^{2}$}

${ }^{1}$ School of Computing, University of Buckingham, Hunter Street, Buckingham, MK18 1EG, United Kingdom, and Centre for Quantum Geometry of Moduli Spaces, Aarhus University, Ny Munkegade 118, Bldg 1530, 8000 Aarhus, Denmark, thomas.madsen@buckingham.ac.uk and ${ }^{2}$ Department of Mathematics, Centre for Quantum Geometry of Moduli Spaces, and Aarhus University Centre for Digitalisation, Big Data and Data Analytics, Aarhus University, Ny Munkegade 118, Bldg 1530, 8000 Aarhus, Denmark, swann@math.au.dk

Correspondence to be sent to: swann@math.au.dk

\begin{abstract}
We study Spin(7)-manifolds with an effective multi-Hamiltonian action of a four-torus. On an open dense set, we provide a Gibbons-Hawking type ansatz that describes such geometries in terms of a symmetric $4 \times 4$-matrix of functions. This description leads to the first known Spin(7)-manifolds with a rank 4 symmetry group and full holonomy. We also show that the multi-moment map exhibits the full orbit space topologically as a smooth four-manifold, containing a trivalent graph in $\mathbb{R}^{4}$ as the image of the set of the special orbits.
\end{abstract}

\section{Introduction}

It was Berger [1] who first realised that the Lie group $\operatorname{Spin}(7)$ could potentially arise as the holonomy group of a non-symmetric irreducible Riemannian manifold. A decade later, Bonan [2] showed that such manifolds would necessarily be Ricci-flat and come with a parallel 4-form of a certain algebraic type. Subsequently the understanding that parallelness amounts to closedness (cf. [8]) has been a powerful tool when looking for examples. The first 8-manifolds with holonomy equal to $\operatorname{Spin}(7)$ were constructed in the late 1980s [3, 4] by Bryant and Salamon; since then many examples have followed, including compact ones by Joyce [12, 13], and most recently infinitely many complete examples by Foscolo [9]. In fact, a torsion-free Spin(7)-structure can be obtained from any closed, spin manifold of dimension 7 [6, Corollary 1.9], but in general this will neither be complete nor have full holonomy.

Received 1 Month 20XX; Revised 11 Month 20XX; Accepted 21 Month 20XX

Communicated by A. Editor

(C) The Author ????. Published by Oxford University Press. All rights reserved. For permissions,

please e-mail: journals.permissions@oxfordjournals.org. 
Despite considerable advances in the field, it still remains a challenge to construct new complete examples with holonomy equal to $\operatorname{Spin}(7)$. A natural way to approach this problem systematically is to consider examples with a specific type of symmetry. Indeed, a key point in constructing the first examples was to apply cohomogeneity one symmetry techniques, and in [15] the first author gave a description of Spin(7)-manifolds with $T^{3}$-symmetry (these are characterised in terms of certain tri-symplectic 4-manifolds).

From a toric viewpoint, it is natural to consider Spin(7)-manifolds with a multi-Hamiltonian action of $T^{4}$, this the critical rank making the dimensions of the leaf space $M / T^{k}$ and the target space of the multi-moment map match. As we will see, this gives the type of behaviour we expect from toric Ricci-flat geometries (cf. [7, 17]). We introduce the notion of a toric Spin(7)-manifold to be a (torsion-free) $\operatorname{Spin}(7)$-manifold $(M, \Phi)$ that comes with an effective multi-Hamiltonian action of a rank four torus. As we explain in $\S 2$, this implies that we have a multi-moment map $\nu: M \rightarrow \mathbb{R}^{4}$ that exhibits an open dense subset $M_{0} \subset M$ as a principle $T^{4}$ bundle over an open subset $\mathcal{U} \subset \mathbb{R}^{4}$. On this regular part, we derive an analogue of the Gibbons-Hawking ansatz. What is needed in this case is a smooth positive definite section $V \in \Gamma\left(\mathcal{U}, S^{2}\left(\mathbb{R}^{4}\right)\right)$ satisfying a pair of PDEs. One of these is a divergence-free condition and the other is a quasi-linear elliptic second order PDE. These equations are natural when one considers differential operators that are invariant, up to scaling, under the GL(4, $\mathbb{R})$ action resulting from changing the basis of the Lie algebra $\mathfrak{t}^{4}$ of the torus $T^{4}$.

In order to achieve a complete understanding of $M$, we address the behaviour near singular orbits in $\S 4$. It turns out that the only special orbits are circles and two-tori. Describing the flat model associated with each singular orbit enables us to show that the orbit space $M / T^{4}$ is homeomorphic to a smooth 4 -manifold, with a global local homeomorphism induced by the multi-moment map $\nu$. It also follows that the image of the singular orbits in $M / T^{4}$ consists of trivalent graphs in $\mathbb{R}^{4}$.

Whilst there are currently no known complete full holonomy Spin(7)-manifolds with a rank four symmetry group, our approach produces the first known incomplete examples, see $§ 5$.

\section{Multi-Hamiltonian Spin(7)-manifolds}

Let $M$ be a connected 8-manifold. A $\operatorname{Spin}(7)$-structure on $M$ is determined by a 4 -form $\Phi$ that is pointwise linearly equivalent to the form $\Phi_{0}=e^{0} \wedge \varphi_{0}+*_{\varphi_{0}} \varphi_{0}$, where

$$
\varphi_{0}=e^{123}-e^{1}\left(e^{45}+e^{67}\right)-e^{2}\left(e^{46}+e^{75}\right)-e^{3}\left(e^{47}+e^{56}\right)
$$

$E_{0}, \ldots, E_{7}$ is a basis of $V \cong \mathbb{R}^{8}, e^{0}, \ldots, e^{7}$ is its dual basis of $V^{*}$, and $e^{123}=e^{1} \wedge e^{2} \wedge e^{3}$, etc. Occasionally, we shall refer to the basis $E_{0}, \ldots, E_{7}$ (and its dual) as being adapted.

The $\mathrm{GL}(V)$-stabiliser of $\Phi_{0}$ is the compact 21-dimensional Lie group $\operatorname{Spin}(7) \subset \mathrm{SO}(V)$. In fact, $\Phi_{0}$ uniquely determines both the inner product $g_{0}=\sum_{j=0}^{7} e_{j}^{2}$ and the volume element $\operatorname{vol}_{0}=e^{01234567}$ (see [3, 14]). Correspondingly, $\Phi$ determines a metric $g$ and a volume form on $M$. 
Following standard terminology, we say that $(M, \Phi)$ is a $\operatorname{Spin}(7)$-manifold if the $\operatorname{Spin}(7)$-structure is torsionfree, hence the (restricted) holonomy group $\operatorname{Hol}_{0}(g)$ is contained in $\operatorname{Spin}(7) \subset \mathrm{SO}(8)$. This implies $g$ is Ricci-flat. It is well-known [8] that being torsion-free, in this context, is equivalent to the condition that $\Phi$ is closed.

We are interested in $\operatorname{Spin}(7)$-manifolds that come with an effective action of a four-torus $T^{4}$ on $M$ that preserves $\Phi$, hence also the metric $g$. This furnishes a Lie algebra homomorphism

$$
\xi: \mathbb{R}^{4} \cong \mathfrak{t}^{4} \rightarrow \mathfrak{X}(M) .
$$

In the following, vector fields in the image of $\xi$ will be said to be generated by the $T^{4}$-action, and we shall occasionally use $\xi_{p}$ to denote the image of $\xi$ at $p \in M$, which is a subspace of $T_{p} M$ of dimension at most 4 .

Definition 2.1 ([16, Def. 3.5]). Let $N$ be a manifold equipped with a closed 4-form $\alpha$, and $G$ an Abelian Lie group acting on $N$ preserving $\alpha$. A multi-moment map for this action is an invariant map $\nu: N \rightarrow \Lambda^{3} \mathfrak{g}^{*}$ such that

$$
d\langle\nu, W\rangle=\xi(W)\lrcorner \alpha,
$$

for all $W \in \Lambda^{3} \mathfrak{g}$; here $\xi(W) \in \Gamma\left(\Lambda^{3} T M\right)$ is the unique multi-vector determined by $W$ via $\xi$.

The $T^{4}$-action being multi-Hamiltonian for $\Phi$ implies that $\left.\Phi\right|_{\Lambda^{4} \xi} \equiv 0$ (cf. [17, Lemma 2.5]), thus $\Phi$ is zero on the tangent spaces of each $T^{4}$-orbit. Let $M_{0}$ be the open dense set of points where the action is free. For $p \in M_{0}$, consider an orthonormal $X_{0}, X_{1}, X_{2}, X_{3} \in \xi_{p}$ with $\hat{\theta}_{i}, i=0,1,2,3$ dual to $X_{0}, X_{1}, X_{2}, X_{3}: \hat{\theta}_{i}\left(X_{j}\right)=\delta_{i j}$ and $\hat{\theta}_{i}(X)=0$ for $X \in\left\langle X_{0}, X_{1}, X_{2}, X_{3}\right\rangle^{\perp}$. Next, let us denote by $\alpha_{i}$ the 1 -forms

$$
\left.\alpha_{i}=(-1)^{i} X_{j} \wedge X_{k} \wedge X_{\ell}\right\lrcorner \Phi,
$$

where $(i j k \ell)=(0123)$ as cyclic permutations.

As $\operatorname{Spin}(7)$ acts transitively on the sphere $S^{7}$, we may take $X_{0}=E_{0}$ at $p$. Now $\left.\varphi=X_{0}\right\lrcorner \Phi$ is a $\mathrm{G}_{2}$-form, isotropic for $X_{1}, X_{2}, X_{3}$. Our analysis of $\mathrm{G}_{2}$-forms $[17, \S 2.2]$ shows that we may take these $X_{i}$ to be $E_{5}, E_{6}, E_{7}$ and so we get:

$$
\Phi=\hat{\theta}_{0} \wedge \varphi+*_{\varphi} \varphi
$$

where

$$
\begin{gathered}
\varphi=\alpha_{123}+\alpha_{1}\left(\alpha_{0} \hat{\theta}_{1}-\hat{\theta}_{23}\right)+\alpha_{2}\left(\alpha_{0} \hat{\theta}_{2}-\hat{\theta}_{31}\right)+\alpha_{3}\left(\alpha_{0} \hat{\theta}_{3}-\hat{\theta}_{12}\right), \\
* \varphi=\hat{\theta}_{123} \alpha_{0}+\alpha_{23}\left(\alpha_{0} \hat{\theta}_{1}-\hat{\theta}_{23}\right)+\alpha_{31}\left(\alpha_{0} \hat{\theta}_{2}-\hat{\theta}_{31}\right)+\alpha_{12}\left(\alpha_{0} \hat{\theta}_{3}-\hat{\theta}_{12}\right),
\end{gathered}
$$

with the shorthand $\alpha_{123}=\alpha_{1} \wedge \alpha_{2} \wedge \alpha_{3}$, and so forth.

Examining the possible isotropy groups, we have the following surprisingly clean result. 
Lemma 2.2. Suppose $T^{4}$ acts effectively on a manifold $M$ with $\operatorname{Spin}(7)$-structure $\Phi$ so that the orbits are isotropic, $\left.\Phi\right|_{\Lambda^{4} \xi_{p}}=0$. Then each isotropy group is connected and of dimension at most two; hence trivial, a circle or $T^{2}$.

Proof. As Spin(7) has rank 3, an isotropy group for $T^{k}$ is of dimension at most 3 . It follows that the $T^{4}$-orbits are at least one-dimensional. In particular, there is always one isotropy invariant direction. Hence, the isotropy group is a subgroup of $\mathrm{G}_{2}$. But $\mathrm{G}_{2}$ has rank 2, so the isotropy group is at most 2-dimensional. Now as in [17, Lemma 2.6], the isotropy group is seen to be a maximal torus in $\mathrm{SU}(r), r=1,2,3$, so is connected and either trivial, a circle or $T^{2}$, as asserted.

In particular, we have that $M_{0}$ is the total space of a principal $T^{4}$-bundle.

\section{Toric $\operatorname{Spin}(7)$-manifolds: local characterisation}

Following the discussion in $\S 2$, we introduce the following terminology:

Definition 3.1. A toric $\operatorname{Spin}(7)$-manifold is a torsion-free $\operatorname{Spin}(7)$-manifold $(M, \Phi)$ with an effective multiHamiltonian action of $T^{4}$.

The main aim of this section is to derive a Gibbons-Hawking type ansatz [10, 11] for toric Spin(7)-manifolds: we obtain a local form for a toric $\operatorname{Spin}(7)$-structure on $M_{0}$ and characterise the torsion-free condition in these terms.

So assume $(M, \Phi)$ is a toric $\operatorname{Spin}(7)$-manifold. Let $U_{0}, U_{1}, U_{2}, U_{3}$ be infinitesimal generators for the $T^{4}$-action; these give a basis for $\xi_{p} \leqslant T_{p} M$ for each $p \in M_{0}$. Denote by $\theta=\left(\theta_{0}, \theta_{1}, \theta_{2}, \theta_{3}\right)^{t}$ the dual basis of $\xi_{p}^{*} \leqslant T_{p}^{*} M$ :

$$
\theta_{i}\left(U_{j}\right)=\delta_{i j} \quad \text { and } \quad \theta(X)=0 \text { for all } X \perp U_{0}, U_{1}, U_{2}, U_{3}
$$

Let $\nu=\left(\nu_{0}, \nu_{1}, \nu_{2}, \nu_{3}\right)^{t}$ be the associated multi-moment map; its components satisfy

$$
\left.d \nu_{i}=(-1)^{i} U_{j} \wedge U_{k} \wedge U_{\ell}\right\lrcorner \Phi=(-1)^{i}\left(U_{j} \times U_{k} \times U_{\ell}\right)^{b}, \quad \text { whenever }(i j k \ell)=(0123),
$$

where the triple cross product $A \times B \times C$ is defined by $g(A \times B \times C, D)=\Phi(A, B, C, D)$ for each $A, B, C, D \in$ $T_{p} M$. It follows that $d \nu$ has full rank on $M_{0}$ and induces a local diffeomorphism $M_{0} / T^{4} \rightarrow \mathbb{R}^{4}$. We define a $4 \times 4$-matrix $B$ of inner products given by

$$
B_{i j}=g\left(U_{i}, U_{j}\right)
$$

On $M_{0}$ we set $V=B^{-1}=\operatorname{det}(B)^{-1} \operatorname{adj}(B)$.

Using the above notation, we have the following local expression for the Spin(7)-structure: 
Proposition 3.2. On $M_{0}$, the 4 -form $\Phi$ is

$$
\begin{gathered}
\Phi=\operatorname{det}(V) \underset{i j k \ell}{\mathfrak{S}}(-1)^{i} \theta_{i} \wedge d \nu_{j k \ell}+\mathfrak{S}_{i j k \ell}(-1)^{\ell} \theta_{i j k} \wedge d \nu_{\ell} \\
+\frac{1}{2 \operatorname{det}(V)}\left(d \nu^{t} \operatorname{adj}(V) \theta\right)^{2} .
\end{gathered}
$$

The associated Spin(7)-metric is given by

$$
g=\frac{1}{\operatorname{det}(V)} \theta^{t} \operatorname{adj}(V) \theta+d \nu^{t} \operatorname{adj}(V) d \nu
$$

Proof. We start by choosing an auxiliary symmetric matrix $A>0$ such that $A^{2}=B^{-1}=V$ which is possible as $B$ is positive definite. Then we set $X_{i}=\sum_{j=0}^{3} A_{i j} U_{j}$ and observe that

$$
g\left(X_{i}, X_{j}\right)=(A B A)_{i j}=\left(A^{2} B\right)_{i j}=\delta_{i j}
$$

showing that the quadruplet $\left(X_{0}, X_{1}, X_{2}, X_{3}\right)$ is orthonormal. It follows that we can apply the formula (2) for $\Phi$. Explicitly,

$$
\begin{aligned}
\Phi=\hat{\theta}_{0} & \wedge \alpha_{123}-\hat{\theta}_{1} \wedge \alpha_{230}+\hat{\theta}_{2} \wedge \alpha_{301}-\hat{\theta}_{3} \wedge \alpha_{012} \\
& +\hat{\theta}_{123} \wedge \alpha_{0}-\hat{\theta}_{230} \wedge \alpha_{1}+\hat{\theta}_{301} \wedge \alpha_{2}-\hat{\theta}_{012} \wedge \alpha_{3} \\
& -\hat{\theta}_{01} \wedge \alpha_{01}-\hat{\theta}_{02} \wedge \alpha_{02}-\hat{\theta}_{30} \wedge \alpha_{30} \\
& -\hat{\theta}_{23} \wedge \alpha_{23}-\hat{\theta}_{31} \wedge \alpha_{31}-\hat{\theta}_{12} \wedge \alpha_{12}
\end{aligned}
$$

We make the identification $\mathbb{R}^{4} \cong \Lambda^{3} \mathbb{R}^{4}$ via contraction with the standard volume form. Then by letting $\Lambda^{3} A$ denote the induced action of $A$ on $\Lambda^{3} \mathbb{R}^{4}$, we get the identity

$$
\Lambda^{3} A=\operatorname{det}(A) A^{-1}
$$

As a result, we get

$$
\alpha=\left(\Lambda^{3} A\right) d \nu \quad \text { and } \quad \hat{\theta}=A^{-1} \theta=\frac{1}{\operatorname{det}(A)}\left(\Lambda^{3} A\right) \theta
$$

The asserted formula for $\Phi$ then follows as the first line of $(4)$ equals $\operatorname{det}(V) \mathfrak{S}_{i j k \ell}(-1)^{i} \theta_{i} \wedge d \nu_{j k \ell}$, the second line reads $\mathfrak{S}_{i j k \ell}(-1)^{\ell} \theta_{i j k} \wedge d \nu_{\ell}$, and the third and fourth lines may be expressed as $\frac{1}{2 \operatorname{det}(V)}\left(d \nu^{t} \operatorname{adj}(V) \theta\right)^{2}$. 
Now the expression from the metric follows by direct computation:

$$
\begin{aligned}
g & =\hat{\theta}^{t} \hat{\theta}+\alpha^{t} \alpha=\left(A^{-1} \theta\right)^{t} A^{-1} \theta+\left(\Lambda^{3} A d \nu\right)^{t} \Lambda^{3} A d \nu \\
& =\theta^{t}\left(\frac{1}{\operatorname{det}(V)} \operatorname{adj}(V)\right) \theta+d \nu^{t} \operatorname{adj}(V) d \nu
\end{aligned}
$$

We remark that there is a natural action of $\mathrm{GL}(4, \mathbb{R})$, corresponding to changing basis of $\mathfrak{t}^{4}$. This action can be useful when looking for invariants, up to scaling, and may also be used to simplify arguments as it allows us to assume that $V$ is diagonal or the identity matrix at a given point, assuming only the $\mathbb{R}^{4}=\widetilde{T^{4}}$ action is of relevance.

\subsection{The torsion-free condition}

The Spin(7)-structure featuring in Proposition 3.2 is generally not torsion-free. To address this, we need to compute $d \Phi$, which involves determining the exterior derivative of $\theta$. By our observations in $\S 2$, we may think of $\theta$ as a connection 1-form and its exterior derivative

$$
d \theta=\omega=\left(\omega_{0}, \omega_{1}, \omega_{2}, \omega_{3}\right)^{t}
$$

is therefore a curvature 2-form (and so represents an integral cohomology class). In terms of our parameterisation for the base space, via the multi-moment map, we can express the curvature components of $\omega$ as

$$
\omega_{a}=\sum_{0 \leqslant i<j \leqslant 3} z_{a}^{i j} d \nu_{i j} .
$$

We collect the curvature coefficients in four skew $4 \times 4$ matrices $Z_{a}=\left(z_{a}^{i j}\right), a=0,1,2,3$. Details of the following calculations may by found in the Appendix.

Closedness of $\Phi$ implies that the curvature matrices $Z_{a}$ are determined via $V$ and $d V$. One gets 28 equations for the 24 variables $z_{a}^{i j}$, the four extra equations reduce to

$$
\sum_{i=0}^{3} \frac{\partial V_{i j}}{\partial \nu_{i}}=0, \quad j=0,1,2,3
$$

We refer to this first order underdetermined elliptic PDE system as the "divergence-free" condition.

The explicit expressions for the curvature coefficients are

$$
z_{a}^{i j}=\sum_{p=0}^{3} V_{p k} \frac{\partial V_{a \ell}}{\partial \nu_{p}}-V_{p \ell} \frac{\partial V_{a k}}{\partial \nu_{p}}
$$


for $k \ell=* i j$, meaning that $d \nu_{k} \wedge d \nu_{\ell}=*_{4} d \nu_{i} \wedge d \nu_{j}$, where $*_{4}$ is with respect to a four-metric for which $d \nu_{i}$ are orthonormal with volume form $d \nu_{0123}$.

There are exactly 10 additional equations, arising from the condition $d \omega=0$. Using (5), these equations can be expressed in the form of a second order quasilinear elliptic PDE without zeroth order terms:

$$
L(V)+Q(d V)=0 .
$$

In the above, the operator $L$ is given by:

$$
L=\sum_{i, j=0}^{3} V_{i j} \frac{\partial^{2}}{\partial \nu_{i} \partial \nu_{j}} .
$$

So $L$ has the same principal symbol as the Laplacian for the metric $d \nu^{t} B d \nu$, which is conformally the same as the restriction of the $\operatorname{Spin}(7)$-metric (3) to the horizontal space. The operator $Q$ is the quadratic form in $d V$ that is given explicitly by

$$
Q(d V)_{i j}=-\sum_{a, b=0}^{3} \frac{\partial V_{i a}}{\partial \nu_{b}} \frac{\partial V_{j b}}{\partial \nu_{a}} .
$$

To summarise, we see that the torsion-free condition determines the curvature matrices $Z_{\ell}$ together with four first order equations and ten second order equations. Hence, we have the following way to locally characterise toric Spin(7)-manifolds.

Theorem 3.3. Any toric Spin(7)-manifold can be expressed in the form of Proposition 3.2 on the open dense subset of principal orbits for the $T^{4}$-action.

Conversely, suppose we are given a principal $T^{4}$-bundle over an open subset $\mathcal{U} \subset \mathbb{R}^{4}$, parameterised by $\nu=\left(\nu_{0}, \nu_{1}, \nu_{2}, \nu_{3}\right)$, together with $V \in \Gamma\left(\mathcal{U}, S^{2}\left(\mathbb{R}^{4}\right)\right)$ that is positive definite at each point. Then the total space comes equipped with a $\operatorname{Spin}(7)$-structure of the form given in Proposition 3.2. This structure is torsion-free, hence toric, if and only if the curvature matrices $Z_{\ell}$ are determined by $V$ via (6), respectively, and $V$ satisfies the divergence-free condition (5) together with the quasilinear second order elliptic system (7).

To conclude this section, we remark that it is possible to integrate the divergence-free equations (5) to obtain a potential. However, the correspondence is not elliptic.

Proposition 3.4. Assume that $V \in \Gamma\left(\mathcal{U}, S^{2}\left(\mathbb{R}^{4}\right)\right)$ satisfies the divergence-free equations (5), with $\mathcal{U} \subset \mathbb{R}^{4}$ simply connected. Then there exists a matrix function $A \in \Gamma\left(\mathcal{U}, M_{6}(\mathbb{R})\right)$ whose second derivatives determine $V$. More precisely, let us index $\mathbb{R}^{6}=\Lambda^{2} \mathbb{R}^{4}$ by $i j=i \wedge j$, for $i, j \in\{0,1,2,3\}$, and write $* i j=k \ell$ as we did after equation (6). Then there is an $A_{i j, k \ell}$ satisfying $A_{i j, * k \ell}=A_{k \ell, * i j}$ such that

$$
V_{a b}=\sum_{k, \ell=0}^{3} \frac{\partial^{2} A_{a k, * b \ell}}{\partial \nu_{k} \partial \nu_{\ell}} .
$$


Proof. We begin by noting that the divergence-free equation can be written more concisely as

$$
d *_{4}(V d \nu)=0
$$

where $\nu=\left(\nu_{0}, \nu_{1}, \nu_{2}, \nu_{3}\right)^{t}$ and $*_{4}$ is the flat Hodge star operator with respect to the $\nu$-coordinates. As $\mathcal{U}$ is simply connected, we deduce that $*_{4} V d \nu$ is exact, i.e.,

$$
V d \nu=*_{4} d(W \kappa)
$$

for some $W \in \Gamma\left(\mathcal{U}, M_{4 \times 6}(\mathbb{R})\right)$ and $\kappa=\left(d \nu_{01}, d \nu_{02}, \ldots, d \nu_{23}\right)^{t}$.

Now, using the symmetry of $V$, we find that $\widetilde{W} \in \Gamma\left(\mathcal{U}, M_{6 \times 4}(\mathbb{R})\right)$ given by $\widetilde{W}_{p q, i}=W_{q, * p i}-W_{p, * q i}$ satisfies:

$$
d *_{4}(\widetilde{W} d \nu)=0
$$

Note $2 W_{i, * p q}=\widetilde{W}_{p q, i}-\widetilde{W}_{q i, p}-\widetilde{W}_{i p, q}$, so $\widetilde{W}$ determines $W$ uniquely. As before, the differential equation can be integrated. Indeed, we can find a section $A \in \Gamma\left(\mathcal{U}, M_{6}(\mathbb{R})\right)$ such that $\widetilde{W} d \nu=*_{4} d(A \kappa)$.

In conclusion, $V$ can be expressed in terms of the second derivatives of the entries of $A$, with the explicit expressions given by (8).

\subsection{Natural PDEs}

We have already remarked that in our description of toric $\operatorname{Spin}(7)$-manifolds there is an action of $\mathrm{GL}(4, \mathbb{R})$ corresponding to a different choice of generators for $\mathfrak{t}^{4} \cong \mathbb{R}^{4}$. As for toric $\mathrm{G}_{2}$-manifolds (cf. [17, $\left.\S 3.2\right]$ ), this action furnishes a way of approaching equation (7), by understanding how the operators $L$ and $Q$ transform under $\operatorname{GL}(4, \mathbb{R})$.

It is useful use the identification $\operatorname{GL}(4, \mathbb{R}) \cong\left(\mathbb{R}^{\times} \times \operatorname{SL}(4, \mathbb{R})\right) / \mathbb{Z}_{2}$, where $\mathbb{Z}_{2}$ is generated by $-1_{4}$, and accordingly express irreducible representations as $\ell^{k} \Gamma_{a, b, c}$, where $\Gamma_{a, b, c}$ is an irreducible representation of $\mathrm{SL}(4, \mathbb{R})$, and $\ell$ is the standard one-dimensional representation of $\mathbb{R}^{\times} \rightarrow \mathbb{R} \backslash\{0\}: t \mapsto t$. As an example, this means that we have for $p \in M_{0}$ that $\xi_{p}=\ell^{1} \Gamma_{0,0,1}$.

Now let $U=\left(\mathbb{R}^{4}\right)^{*}=\ell^{-1} \Gamma_{1,0,0}$, viewed as a representation of $\operatorname{GL}(4, \mathbb{R})$. Then $V \in S^{2}(U)=\ell^{-2} \Gamma_{2,0,0}$. The collection of first order partial derivatives $V^{(1)}=\left(V_{i j, k}\right)=\left(\partial V_{i j} / \partial \nu_{k}\right)$ is then an element of $S^{2}(U) \otimes \ell^{-4} U^{*}=$ $\ell^{-5} \Gamma_{2,0,0} \otimes \Gamma_{0,0,1}$, since $d \nu$ transforms as an element of $\Lambda^{3} U^{*}=\ell^{3} \Gamma_{1,0,0}=\ell^{4} U$. This tensor product decomposes as

$$
S^{2}(U) \otimes \ell^{-4} U^{*}=\ell^{-5} \Gamma_{1,0,0} \oplus \ell^{-5} \Gamma_{2,0,1}
$$

with the projection to $\Gamma_{1,0,0}$ being given by the contraction $S^{2}\left(\Gamma_{1,0,0}\right) \otimes \Gamma_{0,0,1} \rightarrow \Gamma_{1,0,0}$, and $\Gamma_{2,0,1}$ denoting the kernel of this map. The divergence-free equation (5) simply says that this contraction is zero, and so $V^{(1)} \in \ell^{-5} \Gamma_{2,0,1}$. 
The operator $Q$ is a symmetric quadratic operator on $V^{(1)}$ with values in $S^{2}(U)$. Hence, we may think of $Q(d V)$ as an element of the space $\ell^{8} S^{2}\left(\Gamma_{2,0,1}\right)^{*} \otimes S^{2}\left(\Gamma_{1,0,0}\right)$. This space contains exactly one submodule that is trivial as an $\mathrm{SL}(4, \mathbb{R})$-module, since $S^{2}\left(\Gamma_{1,0,0}\right)^{*}$ is a submodule of $S^{2}\left(\Gamma_{2,0,1}\right)^{*}$. Direct computations show that $Q(d V)$ belongs to $\ell^{8}$.

In a similar way, we can address the second order terms in (7). We have $V^{(2)}=\left(V_{i j, k \ell}\right) \in R=\left(S^{2}(U) \otimes\right.$ $\left.S^{2}\left(\ell^{-4} U^{*}\right)\right) \cap\left(\ell^{-8} \Gamma_{2,0,1} \otimes \Gamma_{0,0,1}\right)=\ell^{-8} \Gamma_{1,0,1}+\ell^{-8} \Gamma_{2,0,2}$. Now, $L(V)$ is built from a product of $V$ with $V^{(2)}$ and takes values in $S^{2}(U)$. So $L(V) \in S^{2}(U)^{*} \otimes R^{*} \otimes S^{2}(U)$. In this case, there are two submodules isomorphic to $\ell^{8}$, but only one that appears in $L(V)$, corresponding to the contractions

$$
S^{2}\left(U^{*}\right) \otimes\left(S^{2}\left(U^{*}\right) \otimes S^{2}\left(\ell^{4} U\right)\right) \otimes S^{2}(U) \rightarrow \ell^{8} .
$$

Contracting in this way seems to be the more natural choice.

Summing up the above discussion, $L$ and $Q$ are preserved up to scale by the $G L(4, \mathbb{R})$ change of basis, and this specifies $Q$ uniquely. This is completely analogous to what happens in the $\mathrm{G}_{2}$ setting (see [17, Prop. 3.7]).

Proposition 3.5. Under the action of $\mathrm{GL}(4, \mathbb{R}), L(V)$ and $Q(d V)$ transform as elements of $\ell^{8}$. Moreover, up to scaling, $Q$ is the unique $S^{2}(U)$-valued quadratic form in $d V$ with this property.

\section{Behaviour near singular orbits}

We now want to address the singular behaviour of toric Spin(7)-manifolds. As non-trivial stabilisers, we have tori of dimension 2 or 1 .

For a two-dimensional stabiliser, the flat model is $M=T^{2} \times \mathbb{C}^{3}$, with local coordinates $(x, y)$ on $T^{2}=\mathbb{R}^{2} / \Gamma$ for some lattice $\Gamma, z_{j}=x_{j}+i y_{j}$ on $\mathbb{C}^{3}$. Putting $e^{0}=d x$, and using the standard $\varphi$ on $S^{1} \times \mathbb{C}^{3}$ as in [17], we have

$$
\begin{aligned}
\Phi=\frac{i}{2} d x & \wedge d y \wedge\left(d z_{1} \wedge d \bar{z}_{1}+d z_{2} \wedge d \bar{z}_{2}+d z_{3} \wedge d \bar{z}_{3}\right) \\
+d x & \wedge \operatorname{Re}\left(d z_{1} \wedge d z_{2} \wedge d z_{3}\right) \\
-d y & \wedge \operatorname{Im}\left(d z_{1} \wedge d z_{2} \wedge d z_{3}\right)-\frac{1}{8}\left(d z_{1} \wedge d \bar{z}_{1}+d z_{2} \wedge d \bar{z}_{2}+d z_{3} \wedge d \bar{z}_{3}\right)^{2}
\end{aligned}
$$

with Killing vector fields

$$
\begin{aligned}
U_{0}=\frac{\partial}{\partial x}, \quad U_{1} & =\frac{\partial}{\partial y}, \quad U_{2}=2 \operatorname{Re}\left(i\left(z_{1} \frac{\partial}{\partial z_{1}}-z_{3} \frac{\partial}{\partial z_{3}}\right)\right), \\
U_{3} & =2 \operatorname{Re}\left(i\left(z_{2} \frac{\partial}{\partial z_{2}}-z_{3} \frac{\partial}{\partial z_{3}}\right)\right)
\end{aligned}
$$


generating the $T^{4}$ action. The components of the corresponding multi-moment map are:

$$
\begin{gathered}
\nu_{0}=\operatorname{Im}\left(z_{1} z_{2} z_{3}\right), \quad \nu_{1}=\operatorname{Re}\left(z_{1} z_{2} z_{3}\right) \\
\nu_{2}=-\frac{1}{2}\left(\left|z_{2}\right|^{2}-\left|z_{3}\right|^{2}\right), \quad \nu_{3}=\frac{1}{2}\left(\left|z_{1}\right|^{2}-\left|z_{3}\right|^{2}\right) .
\end{gathered}
$$

For one-dimensional stabiliser the flat model is $M=\left(T^{3} \times \mathbb{R}\right) \times \mathbb{C}^{2}$, with local coordinates $x_{1}, x_{2}, x_{3}, u$ for $T^{3} \times \mathbb{R}$ and $(z, w)$ for $\mathbb{C}^{2}$,

$$
\begin{aligned}
\Phi=d x_{1} & \wedge d x_{2} \wedge d x_{3} \wedge d u \\
& +\left(d x_{2} \wedge d x_{3}-d x_{1} \wedge d u\right) \wedge \frac{i}{2}(d z \wedge d \bar{z}+d w \wedge d \bar{w}) \\
& -d x_{1} \wedge \operatorname{Re}\left(\left(d x_{2}-i d x_{3}\right) \wedge d z \wedge d w\right) \\
& +d u \wedge \operatorname{Im}\left(\left(d x_{2}-i d x_{3}\right) \wedge d z \wedge d w\right) \\
& +\frac{1}{8}(d z \wedge d \bar{z}+d w \wedge d \bar{w})^{2}
\end{aligned}
$$

with vector fields

$$
\begin{gathered}
U_{0}=\frac{\partial}{\partial x_{1}}, \quad U_{1}=\frac{\partial}{\partial x_{2}}, \quad U_{2}=\frac{\partial}{\partial x_{3}}, \\
U_{3}=-2 \operatorname{Re}\left(i\left(z \frac{\partial}{\partial z}-w \frac{\partial}{\partial w}\right)\right) .
\end{gathered}
$$

In this case, the multi-moment map $\nu=\left(\nu_{0}, \nu_{1}, \nu_{2}, \nu_{3}\right)$ has

$$
\nu_{0}=\frac{1}{2}\left(|z|^{2}-|w|^{2}\right), \quad \nu_{1}=-\operatorname{Re}(z w), \quad \nu_{2}=-\operatorname{Im}(z w), \quad \nu_{3}=-u
$$

Now let us consider a general $\operatorname{Spin}(7)$-manifold $M$ with multi-Hamiltonian $T^{4}$-action. Suppose $p \in M$ is a point with stabiliser $T^{2}$. As $\operatorname{Spin}(7)$ acts transitively on the space of two-dimensional subspaces in $\mathbb{R}^{8}$, we may identify $T_{p} M$ with $\mathbb{R}^{2} \times \mathbb{C}^{3}=T_{0}\left(T^{2} \times \mathbb{C}^{3}\right)$ in such a way that the $\operatorname{Spin}(7)$-forms agree at this point.

The exponential map of $M$ at $p$ identifies a neighbourhood of $0 \in T_{0}\left(T^{2} \times \mathbb{C}^{3}\right)$ equivariantly with a neighbourhood of $p \in M$. We may then choose our identifications so that $\left(U_{2}\right)_{p}=0=\left(U_{3}\right)_{p},\left(\nabla U_{2}\right)_{p}=$ $\operatorname{diag}(i, 0,-i)$ and $\left(\nabla U_{3}\right)_{p}=\operatorname{diag}(0, i,-i)$, both in $\mathfrak{s u}(3)$. Now note that $\mathfrak{s p i n}(7)$ contains $\mathfrak{s u}(4)$, so we may consider $T_{0}\left(T^{2} \times \mathbb{C}^{3}\right)$ as $\mathbb{C} \times \mathbb{C}^{3}=\mathbb{C}^{4}$. As $\mathfrak{s p i n}(7)$ and $\mathfrak{s u}(4)$ are both of rank three, it follows that the diagonal maximal torus $\mathfrak{t}^{2}$ in $\mathfrak{s u}(3) \subset \mathfrak{s u}(4)$ has as centraliser in $\mathfrak{s p i n}(7)$ the diagonal maximal torus of $\mathfrak{s u}(4)$. For any $U$ generated by the $T^{4}$-action, we have $(\nabla U)_{p}$ is an element of $\mathfrak{s p i n}(7)$ commuting with both $\left(\nabla U_{2}\right)_{p}$ and $\left(\nabla U_{3}\right)_{p}$, cf. $[17, \S 4.2 .1]$. Thus there exist $a, b \in \mathbb{R}$ such that $\left(\nabla\left(U+a U_{2}+b U_{3}\right)\right)_{p}$ is proportional to $\operatorname{diag}(-3 i, i, i, i)$ acting on $\mathbb{C}^{4}=\mathbb{C} \times \mathbb{C}^{3}=T_{0}\left(T^{2} \times \mathbb{C}^{3}\right)$. It follows that we can choose $U_{0}$ and $U_{1}$ so that at $p$ they are orthonormal (but 
not necessarily integral $),\left(\nabla U_{0}\right)_{p}=c \operatorname{diag}(-3 i, i, i, i), c \in \mathbb{R}$, and $\left(\nabla U_{1}\right)_{p}=0$. But we have

$$
0=\left[U_{0}, U_{1}\right]_{p}=\left(\nabla_{U_{0}} U_{1}\right)_{p}-\left(\nabla_{U_{1}} U_{0}\right)_{p}=0+3 c i U_{1}=-3 c U_{0}
$$

which implies that $c=0$. Hence, $\left(\nabla U_{0}\right)_{p}=0$ too.

As in $[17, \S 4.2 .1-2]$ we may compute many covariant derivatives at $p$. In particular, the Killing condition implies $\nabla_{A, B}^{2} U_{i}=-R_{U_{i}, A} B$, so this vanishes if $\left(U_{i}\right)_{p}=0$. Furthermore, at such a zero $\nabla_{A, B, C}^{3} U_{i}=-R_{\nabla_{A} U_{i}, B} C$. It follows that $\nabla^{m} U_{j}$ agrees with the flat model at $p$ for

$$
(m, j) \in(\{0,1\} \times\{0,1\}) \cup(\{0,1,2\} \times\{2,3\})
$$

and is zero for $(m, j) \in(\{1\} \times\{0,1\}) \cup(\{0,2\} \times\{2,3\})$. Thus $\nabla^{m} \nu_{i}$, which is a sum of terms $\Phi\left(\nabla^{m_{1}} U_{j}, \nabla^{m_{2}} U_{k}, \nabla^{m_{3}} U_{\ell}, \cdot\right)$ with $m=1+m_{1}+m_{2}+m_{3}$, agrees with the flat model at $p$ for

$$
(m, i) \in(\{0,1,2,3,4\} \times\{0,1\}) \cup(\{0,1,2,3\} \times\{2,3\})
$$

This exactly matches the degree of agreement we have in the $\mathrm{G}_{2}$-case, and we can apply the analysis of [17, §4.4] (a controlled comparison with the flat model combined with a degree argument) to conclude that the multi-moment map induces a local homeomorphism of the quotient.

Let us now turn to the case when $p \in M$ has a stabiliser of dimension one. Let $U_{3}$ be a generator for the stabiliser $S^{1}$. Let $U_{0}, U_{1}, U_{2}$ be any three vector fields generated by the $T^{4}$-action, with the property that they are orthonormal at $p$. Then the triple-cross product $\left(U_{0} \times U_{1} \times U_{2}\right)_{p}$ is an invariant unit vector in $T_{p} M$ that is orthogonal to the orbit. As $\operatorname{Spin}(7)$ acts transitively on three-dimensional subspaces of $\mathbb{R}^{8}$ we may identify $T_{p} M$ with $\left(\mathbb{R}^{3} \times \mathbb{R}\right) \times \mathbb{C}^{2}=T_{0}\left(T^{3} \times \mathbb{R}\right) \times \mathbb{C}^{2}$ in such a way that $\left(\nabla U_{3}\right)_{p}$ acts as $\operatorname{diag}(i,-i) \in \mathfrak{s u}(2)$ and the $\operatorname{Spin}(7)$-forms agree at $p$. We have $d \nu_{3}=-\left(U_{0} \times U_{1} \times U_{2}\right)^{b}$ is non-zero at $p$ and so provides an invariant transverse coordinate to a seven-dimensional level set through $p$. We have $\left(d \nu_{i}\right)_{p}=0$, for $i=0,1,2$, and $\nabla^{2} \nu_{i}$ is determined at $p$ by $U_{0}, U_{1}, U_{2}$ and $\nabla U_{3}$ via $\Phi$, so these agree with the flat model at this point. This means that we can once again apply the $\mathrm{G}_{2}$-analysis to conclude that the multi-moment maps provide a local homeomorphism to $\mathbb{R}^{4}$ around $p$.

Summarising the discussion of this section, we have the following description of the orbit space of toric $\operatorname{Spin}(7)$-manifolds:

Theorem 4.1. Let $(M, \Phi)$ be a toric Spin(7)-manifold. Then $M / T^{4}$ is homeomorphic to a smooth four-manifold. Moreover, the multi-moment map $\nu$ induces a local homeomorphism $M / T^{4} \rightarrow \mathbb{R}^{4}$.

We suspect that the image of the set of special orbits plays an important role, so it is worthwhile addressing this topic more explicitly. First, if $p \in M$ is a point with stabiliser $S^{1}$, then taking linear combinations of vector 
fields in the above analysis, potentially loosing orthogonality, allows so to find an integral basis $U_{0}, U_{1}, U_{2}, U_{3}$ of $\mathfrak{t}^{4}$ such that $\left(U_{3}\right)_{p}=0$. Inspection shows that $U_{3}$ is zero for all points of $T^{3} \times \mathbb{R}$ in the flat model. Hence, the $\nu_{1}, \nu_{2}$ and $\nu_{3}$ are constant on this set, and the image under $\nu$ of this family of singular orbits is a straight line parameterised by $\nu_{3}$.

Next, let us consider the case when $p$ has $T^{2}$ as its isotropy subgroup. Then the normal bundle of the two-torus $T^{4} p$ is $\mathbb{C}^{3}$, and there are three families of points with circle stabiliser, meeting at $p$. Again looking at the associated flat model, we see that there is an integral basis $U_{0}, U_{1}, U_{2}, U_{3}$ of $\mathfrak{t}^{4}$ that has $\left(U_{2}\right)_{p}=0=\left(U_{3}\right)_{p}$ at $p$ and such that $U_{2}, U_{3}$ and $-U_{2}-U_{3}$ generate the circle stabilisers of the three families. The images of these families under $\nu$ have constant $\nu_{0}$ and $\nu_{1}$ coordinates, and give three half-lines meeting at $\nu(p)$ and lying in $\nu_{2}$, $\nu_{3}$ or $\nu_{2}-\nu_{3}$ constant.

Proposition 4.2. The image in $M / T^{4}$ of the union $M \backslash M_{0}$ of singular orbits consists locally of trivalent graphs in $\mathbb{R}^{4}$ with edges that are straight lines of rational slope in the $\nu$-coordinates, with primitive slope vectors summing to zero at each vertex.

Proof. Fix a choice of integral basis for $\mathfrak{t}^{4}$. Then any other integrable basis is obtained via the action of GL $(4, \mathbb{Z})$ and this group in turn acts on $\left(\nu_{0}, \ldots, \nu_{3}\right)$. In particular, local families of points with stabiliser $S^{1}$ map $\nu$ to a straight line that is the $\mathrm{GL}(4, \mathbb{Z})$-image of a coordinate axis $\mathbb{R}_{3}$, so has rational slope. At the image of points with stabiliser $T^{2}$, three such lines meet and their primitive tangent vectors are the GL(4,Z)-image of $\mathbf{e}_{2}, \mathbf{e}_{3}$ and $\mathbf{e}_{2}-\mathbf{e}_{3}$, so sum to zero.

\section{Orthogonal Killing vectors}

In contrast with the $\mathrm{G}_{2}$-case (see, for example, $[17, \S 5.1 .2]$ ), there are no known examples of complete toric Spin(7)-manifolds with full holonomy. On the other hand, one would expect that also in this setting, the analysis of 'diagonal' solutions might lead to simple explicit metrics with full holonomy. In general, the metrics of full holonomy arising from the considerations below are incomplete. However, our analysis is not exhaustive, so we cannot rule out the existence of complete examples.

So let us assume $V_{i j}=0$ for all $i \neq j$, i.e., the generating vector fields for the torus action are orthogonal. Writing $V_{i}$ for $V_{i i}$, the $\operatorname{Spin}(7)$-metric now takes the form

$$
g=\sum_{i=0}^{3} \frac{1}{V_{i}}\left(\theta_{i}^{2}+V_{0} V_{1} V_{2} V_{3} d \nu_{i}^{2}\right) .
$$


In this case, the curvature 2 -forms associated with the $T^{4}$ fibration are given by:

$$
\begin{aligned}
& \omega_{0}=-V_{3} \frac{\partial V_{0}}{\partial \nu_{3}} d \nu_{12}+V_{2} \frac{\partial V_{0}}{\partial \nu_{2}} d \nu_{13}-V_{1} \frac{\partial V_{0}}{\partial \nu_{1}} d \nu_{23}, \\
& \omega_{1}=V_{3} \frac{\partial V_{1}}{\partial \nu_{3}} d \nu_{02}-V_{2} \frac{\partial V_{1}}{\partial \nu_{2}} d \nu_{03}+V_{0} \frac{\partial V_{1}}{\partial \nu_{0}} d \nu_{23}, \\
& \omega_{2}=-V_{3} \frac{\partial V_{2}}{\partial \nu_{3}} d \nu_{01}+V_{1} \frac{\partial V_{2}}{\partial \nu_{1}} d \nu_{03}-V_{0} \frac{\partial V_{2}}{\partial \nu_{0}} d \nu_{13}, \\
& \omega_{3}=V_{2} \frac{\partial V_{3}}{\partial \nu_{2}} d \nu_{01}-V_{1} \frac{\partial V_{3}}{\partial \nu_{1}} d \nu_{02}+V_{0} \frac{\partial V_{3}}{\partial \nu_{0}} d \nu_{12}
\end{aligned}
$$

The divergence-free condition tells us that $\partial V_{i} / \partial \nu_{i}=0$, for $i=0,1,2,3$. Then the condition $d \omega=0$ is given by the equations

$$
\sum_{j=0}^{3} V_{j} \frac{\partial^{2} V_{i}}{\partial \nu_{j}^{2}}=0, \quad i=0,1,2,3
$$

together with

$$
\frac{\partial V_{i}}{\partial \nu_{j}} \frac{\partial V_{j}}{\partial \nu_{i}}=0, \quad i, j \in\{0,1,2,3\}, i \neq j .
$$

Proposition 5.1. After permuting indices, analytic solutions to equation (10) with $\partial V_{i} / \partial \nu_{i}=0$ for all $i$, have one of the following forms:

1. $V_{0}=V_{0}\left(\nu_{1}, \nu_{2}, \nu_{3}\right), V_{1}=V_{1}\left(\nu_{2}, \nu_{3}\right), V_{2}=V_{2}\left(\nu_{3}\right), V_{3}$ constant;

2. $V_{0}=V_{0}\left(\nu_{1}, \nu_{2}, \nu_{3}\right), V_{1}=V_{1}\left(\nu_{2}\right), V_{2}=V_{2}\left(\nu_{3}\right), V_{3}=V_{3}\left(\nu_{1}\right)$;

3. $V_{0}=V_{0}\left(\nu_{1}, \nu_{3}\right), V_{1}=V_{1}\left(\nu_{2}, \nu_{3}\right), V_{2}=V_{2}\left(\nu_{0}, \nu_{3}\right), V_{3}$ constant;

4. $V_{0}=V_{0}\left(\nu_{1}, \nu_{2}\right), V_{1}=V_{1}\left(\nu_{2}, \nu_{3}\right), V_{2}=V_{2}\left(\nu_{3}\right), V_{3}=V_{3}\left(\nu_{0}\right)$;

Proof. Define $r=r\left(V_{0}, \ldots, V_{3}\right)$ to be the largest number such that some $V_{i}$ has $r$ partial derivatives $\partial V_{i} / \partial \nu_{j}$ not identically zero.

If $r=3$, then we reorder indices so that there is a on open dense set $U$ on which $\left(\partial V_{0} / \partial \nu_{i}\right)_{p} \neq 0$ for $i=1,2,3$ and all $p \in U$. Equation (10) then gives $\partial V_{i} / \partial \nu_{0}=0$ on $U_{0}$ for $i=1,2,3$. Thus $V_{1}=V_{1}\left(\nu_{2}, \nu_{3}\right), V_{2}=V_{2}\left(\nu_{1}, \nu_{3}\right)$ and $V_{3}\left(\nu_{1}, \nu_{2}\right)$. Let $r^{\prime}=r\left(V_{1}, V_{2}, V_{3}\right)$. If $r^{\prime}=2$, then we rearrange to get $\left(\partial V_{1} / \partial \nu_{j}\right)_{p} \neq 0$ for $j=2,3$, for all $p$ in a (smaller) open dense set. It follows that $V_{2}=V_{2}\left(\nu_{3}\right)$ and $V_{3}=V_{3}\left(\nu_{2}\right)$. But by (10), only one of these can have a derivative that is not identically zero, so we have case 1 . If $r^{\prime}=1$, then we may assume $V_{1}=V_{1}\left(\nu_{2}\right)$ is not constant zero. It follows that $\partial V_{2} / \partial \nu_{1} \equiv 0$, so $V_{2}=V_{2}\left(\nu_{3}\right)$ and we get case 2 .

If $r=2$, then we may take $V_{0}=V_{0}\left(\nu_{1}, \nu_{2}\right)$, non-constant in each variable. This implies $V_{1}=V_{1}\left(\nu_{2}, \nu_{3}\right)$. If $V_{1}$ is non-constant in both variables, then $V_{2}=V_{2}\left(\nu_{3}\right)$ and $V_{3}=V_{3}\left(\nu_{0}, \nu_{2}\right)$. Now either $V_{2}$ is constant, which may be rearranged to case 3 , or $V_{3}=V_{3}\left(\nu_{0}\right)$, which is case 4 . 
For $r=1$, we may assume $V_{0}=V_{0}\left(\nu_{1}\right)$. If this is non-constant, then we may take $V_{1}=V_{1}\left(\nu_{2}\right)$. When $V_{1}$ is non-constant, we then have $V_{2}$ is $V_{2}\left(\nu_{3}\right)$ or $V_{2}\left(\nu_{0}\right)$. In the former case $V_{3}=V_{3}\left(\nu_{0}\right)$, a subcase of 2 , or $V_{3}=V_{3}\left(\nu_{1}\right)$, a subcase of 4 . The latter case gives subcases of 2 .

Solutions to the full $\operatorname{Spin}(7)$ equations with $V_{3}$ constant are just Riemannian products of a circle with a toric $\mathrm{G}_{2}$-manifold. Thus irreducible solutions have to fall under cases 2 or 4 above. A simple solution of the form 4 is given by taking $V_{0}=\nu_{1}, V_{1}=\nu_{2}, V_{2}=\nu_{3}, V_{3}=\nu_{0}, \nu_{i}>0$ for all $i$. This gives the following metric for which a curvature computation shows that its (restricted) holonomy is equal to Spin(7):

$$
\begin{aligned}
g= & \frac{1}{\nu_{1}} \theta_{0}^{2}+\frac{1}{\nu_{2}} \theta_{1}^{2}+\frac{1}{\nu_{3}} \theta_{2}^{2}+\frac{1}{\nu_{0}} \theta_{3}^{2} \\
& +\nu_{2} \nu_{3} \nu_{0} d \nu_{0}^{2}+\nu_{1} \nu_{3} \nu_{0} d \nu_{1}^{2}+\nu_{1} \nu_{2} \nu_{0} d \nu_{2}^{2}+\nu_{1} \nu_{2} \nu_{3} d \nu_{3}^{2}
\end{aligned}
$$

where

$$
d \theta_{0}=-\nu_{2} d \nu_{23}, \quad d \theta_{1}=\nu_{3} d \nu_{30}, \quad d \theta_{2}=-\nu_{0} d \nu_{01}, \quad d \theta_{3}=\nu_{1} d \nu_{12}
$$

We claim that the above metric can not be made complete by adding singular orbits. One way to see this is to use the criterion of [5, Lemma 1] which implies incompleteness of $g$ provided we can find a finite length curve that is not contained in any compact set. As the fibres are compact, it is sufficient to find such a curve in the base space of our $T^{4}$-bundle. Now, consider the straight lines with $\nu_{1}, \nu_{2}$ and $\nu_{3}$ constant. Each such curve is parameterised by $\nu_{0} \in \mathbb{R}_{>0}$ and on $[a, b] \subset \mathbb{R}_{>0}$ has length a positive constant times $b^{3 / 2}-a^{3 / 2}$, which is finite as $a \rightarrow 0$. However, the metric $B=V^{-1}$ on the torus orbits satisfies $\operatorname{det}(B)=1 /\left(\nu_{0} \nu_{1} \nu_{2} \nu_{3}\right) \rightarrow \infty$ as $\nu_{0} \rightarrow 0$, so the discussion in Section 4 shows that we are not approaching a singular orbit. Thus the metric is incomplete, and this type of argument applies near any point of the boundary $\prod_{i=0}^{3} \nu_{i}=0$. On the other hand, we have completeness in directions with one or more $\nu_{i} \rightarrow+\infty$ and the other coordinates bounded away from zero, since on the base the metric is bounded below by the flat metric $\sum_{i=0}^{3} d \nu_{i}^{2}$ on $(\mathbb{R})_{>0}^{4}$ which is complete in these directions. The asymptotic behaviour can be read off from the metric expression above, with the number of unbounded $\nu_{i}$ 's corresponding to the dimension of the subtorus that collapses.

Now let us turn to case 2. In general, if $V_{i}$ is a function of a single variable, then equation (9) forces $V_{i}$ to be linear in that variable. Thus after an affine change of variables, for irreducible solutions in case 2 , the equations (9) become

$$
\nu_{2} \frac{\partial^{2} V_{0}}{\partial \nu_{1}^{2}}+\nu_{3} \frac{\partial^{2} V_{0}}{\partial \nu_{2}^{2}}+\nu_{1} \frac{\partial^{2} V_{0}}{\partial \nu_{3}^{2}}=0
$$

A simple solution is then $V_{0}=\nu_{1} \nu_{2} \nu_{3}$ giving the metric

$$
\begin{aligned}
g= & \frac{1}{\nu_{1} \nu_{2} \nu_{3}} \theta_{0}^{2}+\frac{1}{\nu_{2}} \theta_{1}^{2}+\frac{1}{\nu_{3}} \theta_{2}^{2}+\frac{1}{\nu_{1}} \theta_{3}^{2} \\
& +\nu_{1} \nu_{2} \nu_{3} d \nu_{0}^{2}+\nu_{1}^{2} \nu_{2} \nu_{3}^{2} d \nu_{1}^{2}+\nu_{1}^{2} \nu_{2}^{2} \nu_{3} d \nu_{2}^{2}+\nu_{1} \nu_{2}^{2} \nu_{3}^{2} d \nu_{3}^{2}
\end{aligned}
$$


where

$$
\begin{gathered}
d \theta_{0}=-\nu_{1}^{2} \nu_{2} d \nu_{12}-\nu_{3}^{2} \nu_{1} d \nu_{31}-\nu_{2}^{2} \nu_{3} d \nu_{23} \\
d \theta_{1}=-\nu_{3} d \nu_{03}, \quad d \theta_{2}=-\nu_{1} d \nu_{01}, \quad d \theta_{3}=-\nu_{2} d \nu_{02}
\end{gathered}
$$

on $\nu_{i}>0$ for $i=1,2,3$. Another solution is obtained by taking $V_{0}=\nu_{1}^{3} \nu_{3}+\nu_{2}^{3} \nu_{1}-2 \nu_{3}^{3} \nu_{2}$ on the non-empty domain where $V_{0}>0$ and $\nu_{i}>0$ for $i=1,2,3$. Using similar ideas as in the previous example, one sees that this metric is incomplete.

For case 4, we have

$$
V_{1} \frac{\partial^{2} V_{0}}{\partial \nu_{1}^{2}}+\nu_{3} \frac{\partial^{2} V_{0}}{\partial \nu_{2}^{2}}=0, \quad \nu_{3} \frac{\partial^{2} V_{1}}{\partial \nu_{2}^{2}}+\nu_{0} \frac{\partial^{2} V_{1}}{\partial \nu_{3}^{2}}=0
$$

But this holds for an open set in all the variables. In the second equation, letting $\nu_{0}$ vary we see that $V_{1}=V_{1}\left(\nu_{2}, \nu_{3}\right)$ must be linear in each variable, $V_{1}=A+B \nu_{2}+C \nu_{3}+D \nu_{2} \nu_{3}$. Considering the $\nu_{3}$-dependence, the first equation decouples as

$$
\left(A+B \nu_{2}\right) \frac{\partial^{2} V_{0}}{\partial \nu_{1}^{2}}=0, \quad\left(C+D \nu_{2}\right) \frac{\partial^{2} V_{0}}{\partial \nu_{1}^{2}}+\frac{\partial^{2} V_{0}}{\partial \nu_{2}^{2}}=0
$$

If $A$ or $B$ is non-zero, then $V_{0}=V_{0}\left(\nu_{1}, \nu_{2}\right)$ becomes linear in both variables; otherwise we have $V_{1}=C \nu_{3}+D \nu_{2} \nu_{3}$ and $V_{0}$ satisfies the last equation of (12).

\section{Acknowledgements}

AFS was partially supported by the Danish Council for Independent Research | Natural Sciences project DFF

- 6108-00358. TBM is grateful for financial support by University of Buckingham through a Dennison Personal Research Grant. Both authors partially supported by the Danish National Research Foundation grant DNRF95 (Centre for Quantum Geometry of Moduli Spaces - QGM). We thank the referee for helpful comments.

\section{A Appendix: deriving the torsion-free equations}

To derive equations (5), (6), and (7), we begin by rewriting $\Phi$. Expanding $\operatorname{adj}(V)$ in terms of entries of $V$, one finds that

$$
\frac{1}{2 \operatorname{det}(V)}\left(d \nu^{t} \operatorname{adj}(V) \theta\right)^{2}=-\sum_{i<j} \sum_{p<q}\left(\Lambda^{2} V\right)_{* i j, * p q} \theta_{i j} \wedge d \nu_{p q}
$$


where $\Lambda^{2} V$ is the matrix of the action of $V$ on 2-forms. Using this in $\Phi$, we compute

$$
\begin{aligned}
d \Phi=d & (\operatorname{det}(V)) \mathfrak{S}_{i j k \ell}(-1)^{i} \theta_{i} \wedge d \nu_{j k \ell}+\sum_{a, b} \sum_{p<q}\left(\Lambda^{2} V\right)_{* a b, * p q} \theta_{a} \wedge \omega_{b} \wedge d \nu_{p q} \\
& +\mathfrak{S}_{i j k \ell}(-1)^{\ell}\left(\theta_{j k} \wedge \omega_{i}-\theta_{i k} \wedge \omega_{j}+\theta_{i j} \wedge \omega_{k}\right) \wedge d \nu_{\ell} \\
& -\sum_{i<j} \sum_{p<q} \theta_{i j} \wedge d\left(\Lambda^{2} V_{* i j, * p q}\right) \wedge d \nu_{p q}
\end{aligned}
$$

The second line is equal to $-\sum_{i<j} \theta_{i j}\left(\omega_{k} d \nu_{\ell}-\omega_{\ell} d \nu_{k}\right)$, where $k \ell=* i j$. Equating coefficients of $\theta_{i j} \wedge d \nu_{* k}$ in $d \Phi=0$ gives 24 linear equations for the 24 coefficients $z_{a}^{i j}$ of $\left(\omega_{a}\right)_{a=0}^{3}$ that directly give $z_{a}^{i j}$, when $a, i$ and $j$ are distinct, and the simple sums $z_{a}^{a i}+z_{a}^{a j}$, for $i, j \neq a$. Solving these, we find

$$
z_{a}^{i j}=\frac{1}{2}\left(\operatorname{div}\left(\left(\Lambda^{2} V\right)_{k \ell, a} .\right)+\operatorname{div}\left(\left(\Lambda^{2} V\right)_{k a, \ell} .\right)+\operatorname{div}\left(\left(\Lambda^{2} V\right)_{a \ell, k} .\right)\right),
$$

where $k \ell=* i j$, and div denotes divergence: $\operatorname{div}(W)=.\sum_{k=0}^{3} \partial W_{k} / \partial \nu_{k}$, etc. Substituting into the first line of $d \Phi=0$ and extracting the coefficients of $\theta_{i} \wedge d \nu_{0123}$, we get a four-vector. Multiplying this by $V$ gives the four equations $\operatorname{det}(V) \operatorname{div}\left(V_{i}.\right)=0$ and hence the divergence-free equation (5). The expression for $z_{a}^{i j}$ now reduces to $(6)$.

We next turn to the curvature conditions $d \omega_{a}=0$. Using the expressions for the $z_{a}^{i j}$, we have

$$
\begin{aligned}
d \omega_{a} & =\sum_{i<j} \sum_{q} \frac{\partial z_{a}^{i j}}{\partial \nu_{q}} d \nu_{q i j} \\
& =\sum_{i<j} \sum_{p, q}\left(V_{p k} \frac{\partial^{2} V_{a \ell}}{\partial \nu_{p} \partial \nu_{q}}-V_{p \ell} \frac{\partial^{2} V_{a k}}{\partial \nu_{p} \partial \nu_{q}}+\frac{\partial V_{p k}}{\partial \nu_{q}} \frac{\partial V_{a \ell}}{\partial \nu_{p}}-\frac{\partial V_{p \ell}}{\partial \nu_{q}} \frac{\partial V_{a k}}{\partial \nu_{p}}\right) d \nu_{q i j}, \quad \text { where } k \ell=* i j \\
& =-\sum_{b, p} \sum_{k \neq b}\left(V_{p k} \frac{\partial^{2} V_{a b}}{\partial \nu_{p} \partial \nu_{k}}-V_{p b} \frac{\partial^{2} V_{a k}}{\partial \nu_{p} \partial \nu_{k}}+\frac{\partial V_{p k}}{\partial \nu_{k}} \frac{\partial V_{a b}}{\partial \nu_{p}}-\frac{\partial V_{p b}}{\partial \nu_{k}} \frac{\partial V_{a k}}{\partial \nu_{p}}\right) d \nu_{* b} .
\end{aligned}
$$

Inserting the terms $k=b$, so we sum freely over $b, p$, and $k$, does not change the value of this expression. Then, using the divergence-free conditions, we get $d \omega_{a}=-\sum_{b}(L(V)+Q(d V))_{a b} d \nu_{* b}$ and hence the elliptic equation (7).

\section{References}

[1] M. Berger. Sur les groupes d'holonomie homogène des variétés à connexion affine et des variétés riemanniennes. Bull. Soc. Math. France, 83:279-330, 1955.

[2] E. Bonan. Sur des variétés riemanniennes à groupe d'holonomie $G_{2}$ ou $\operatorname{Spin}(7)$. C. R. Acad. Sci. Paris Sér. $A-B, 262: \mathrm{A} 127-\mathrm{A} 129,1966$.

[3] R. L. Bryant. Metrics with exceptional holonomy. Ann. of Math. (2), 126(3):525-576, 1987 . doi: 10.2307/1971360. 
[4] R. L. Bryant and S. M. Salamon. On the construction of some complete metrics with exceptional holonomy. Duke Math. J., 58(3):829-850, 1989. doi: 10.1215/S0012-7094-89-05839-0.

[5] V. Cortés, X. Han, and T. Mohaupt, Completeness in supergravity constructions. Comm. Math. Phys. 311 (1): 191-213, 2012 .

[6] D. Crowley and J. Nordström. New invariants of $G_{2}$-structures. Geom. Topol., 19(5):2949-2992, 2015. doi: 10.2140/gt.2015.19.2949.

[7] A. S. Dancer and A. F. Swann. Hypertoric manifolds and hyperKähler moment maps. In S. Chiossi, A. Fino, E. Musso, F. Podestà, and L. Vezzoni, editors, Special metrics and group actions in geometry, number 23 in Springer INdAM Series, pages 107-127. Springer, Cham, 2017.

[8] M. Fernández. A classification of Riemannian manifolds with structure group Spin(7). Ann. Mat. Pura Appl. (4), 143:101-122, 1986. doi: 10.1007/BF01769211.

[9] L. Foscolo. Complete non-compact Spin(7)-manifolds from self-dual Einstein 4-orbifolds. arXiv:1901.04074 [math.DG], 2019.

[10] G. W. Gibbons and S. W. Hawking. Gravitational multi-instantons. Phys. Lett. B, 78(4):430-432, 1978.

[11] G. W. Gibbons and S. W. Hawking. Classification of gravitational instanton symmetries. Comm. Math. Phys., 66(3):291-310, 1979

[12] D. Joyce. Compact 8-manifolds with holonomy Spin(7). Invent. Math., 123(3):507-552, 1996. doi: $10.1007 / \mathrm{s} 002220050039$.

[13] D. Joyce. A new construction of compact 8-manifolds with holonomy Spin(7). J. Differential Geom., 53 (1):89-130, 1999 .

[14] S. Karigiannis. Deformations of $G_{2}$ and $\operatorname{Spin}(7)$ structures. Canad. J. Math., 57(5):1012-1055, 2005. doi: 10.4153/CJM-2005-039-x.

[15] T. B. Madsen. Spin(7)-manifolds with three-torus symmetry. J. Geom. Phys., 61(11):2285-2292, 2011. doi: 10.1016/j.geomphys.2011.07.008

[16] T. B. Madsen and A. F. Swann. Closed forms and multi-moment maps. Geom. Dedicata, 165:25-52, 2013. doi: 10.1007/s10711-012-9783-4.

[17] T. B. Madsen and A. F. Swann. Toric geometry of $\mathrm{G}_{2}$-manifolds. Geom. Topol., to appear. 